\title{
Diseño de Medidor de Control en el Proceso de Producción de una Planta de Panificación y Repostería
}

\author{
Sánchez-Vásquez Viviana, Profesora Investigadora \\ Caicedo-Camposano Oscar, Profesor Investigador \\ Mora-Castro Oscar, Subdecano Facultad de Ciencias Agropecuarias \\ Espinoza-Espinoza Fernando, Estudiante de Ingeniería Agronómica \\ Tandazo-Garcés Juan, Ingeniero Agrónomo \\ Universidad Técnica de Babahoyo, Ecuador \\ Decker-Campuzano Fernando, Profesor Investigador \\ Universidad Agraria del Ecuador
}

Doi:10.19044/esj.2018.v14n23p155 URL:http://dx.doi.org/10.19044/esj.2018.v14n23p155

\begin{abstract}
The following work was carried out in a baking and confectionery plant, in order to guarantee the quality of the products by optimizing the processes in these facilities, for which the principles set out in the Regulation of Good Practices for Processed Foods were taken into consideration.

It's desired to obtain products with food quality to provide healthy and safe food. For this, we take as a basis the regulations of Good Manufacturing Practices (GMP).

This is how a study was conducted where proposals for key procedures and instructions were raised and executed to identify critical control points for improvement, followed by the implementation of Good Manufacturing Practices in a Bakery line.

Good Manufacturing Practices are the sanitary foundation under which any company related to the processing and handling of food must operate, ensuring that the simplest of operations throughout the process of manufacturing a food product, is carried out under conditions that contribute to the assurance of the quality, hygiene and safety of the product. The GMP is in itself a system of quality control and safety through the elimination and / or reduction of contamination risks of a product.
\end{abstract}

Keywords: Good manufacturing practices, quality control, food safety 


\section{Resumen}

El siguiente trabajo fue realizado en una planta de panificación y repostería, con el propósito de garantizar la calidad de los productos optimizando los procesos en estas instalaciones, para esto se tomó en consideración los principios expuestos en el Reglamento de Buenas Prácticas para alimentos Procesados.

Se desea la obtención de productos con calidad alimentaria para que proporcionar alimentos sanos e inocuos. Para esto tomamos como base los reglamentos de las Buenas Prácticas de Manufactura BPM.

Es así como se realizó un estudio donde se planteó y ejecutaron propuestas de procedimientos e instructivos claves para identificar los puntos críticos de control para su mejora, seguido de la Implementación de Buenas Prácticas de Manufactura en una línea de Panadería.

Las Buenas Prácticas de Manufactura (BPM) constituyen el fundamento sanitario bajo el cual toda empresa relacionada con el procesamiento y el manejo de alimentos debe operar, garantizando que la más sencilla de las operaciones a lo largo del proceso de manufactura de un producto alimenticio, se realice bajo condiciones que contribuyan al aseguramiento de la calidad, higiene y seguridad del producto. Las BPM son en sí un sistema de control de calidad y de seguridad a través de la eliminación y/o reducción de riesgos de contaminación de un producto.

Palabras claves: Buenas prácticas de manufactura, control de calidad, inocuidad alimentaria.

\section{Introducción}

Uno de los principios básicos del Aseguramiento de la Calidad sostiene que un producto debe ser hecho bien desde la primera vez. Esto conlleva a la adopción de un criterio totalmente preventivo en los procesos productivos en lugar de uno correctivo, basado en la inspección final o en el control del producto realizado por el consumidor.

El Aseguramiento de la Calidad se trata de tener bajo control todo el proceso productivo, esto es, desde antes del ingreso de los insumos y materias primas a la empresa (control de proveedores), durante el proceso mismo de producción (documentación y controles en proceso) y después del mismo (control de almacenamiento, transporte y distribución), es decir en cada eslabón de la cadena de producción del producto.

El primer paso hacia la implementación de este tipo de sistemas es la aplicación de ciertos criterios que, aunque mínimos, nos aseguran que los productos son elaborados de manera segura y con una calidad apropiada al uso que se les dará. De esto tratan las Buenas Prácticas de Manufactura (BPM). 
Las Buenas Prácticas de Manufactura (BPM) constituyen el fundamento sanitario bajo el cual toda empresa relacionada con el procesamiento y el manejo de alimentos debe operar, asegurando que hasta la más sencilla de las operaciones a lo largo del proceso de manufactura de un alimento, se realice bajo condiciones que contribuyan al objetivo último de calidad, higiene y seguridad del producto. Las BPM son en sí mismas un sistema de control de calidad y de seguridad a través de la eliminación y/o reducción de riesgos de contaminación de un producto (Sánchez y Decker 2010).

Las exigencias mínimas para que los alimentos sean considerados aptos para el consumo humano es que sean inocuos, saludables y sanos. Para esto es necesario adoptar normas básicas que involucran los cuidados y reglas a seguir por parte de los productores industriales o manipuladores de alimentos, para mantenerse competitivos (Decreto Ejecutivo 3253 2002)

La empresa que pretenda ser competitiva en los mercados globalizados de la actualidad deberá tener una Política de Calidad estructurada a partir de la aplicación de las BPM como punto de partida para la aplicación de sistemas más complejos y de Aseguramiento de la Calidad como es el Análisis de Riesgos y Puntos Críticos de Control (HACCP), la implementación de normas ISO 9000 para llegar finalmente a la Gestión de la Calidad Total (TQM).

Parte de los prerrequisitos para todo programa de Buenas Prácticas de Manufactura es el establecer Procedimientos Estándar de Limpieza y Desinfección (SSOP) que son herramientas esenciales para controlar los riesgos y los Procedimientos Estándar de Operación (SOP) los cuales son métodos para producción de alimentos que reduzcan riesgos a los clientes, ambos son herramientas esenciales para mantener con éxito las Buenas Prácticas de Manufactura.

Los SSOP's son los procedimientos que describen todas aquellas actividades relacionadas con el manejo sanitario de alimentos y la limpieza del ambiente donde se lleva a cabo su producción. Estos procedimientos generan grandes beneficios a la industria alimentaria ya que ayudan a la identificación de tendencias, ayudan a prevenir problemas relacionados con la calidad de los productos, son una herramienta para la capacitación del personal, sirven como evidencia para compradores e inspectores y conducen al mejoramiento continuo de las prácticas y condiciones sanitarias de la industria alimentaria.

En los tiempos que corren existe la preocupación por los alimentos que ingerimos, y es por ello que es muy importante hablar de inocuidad tanto en la producción como en la elaboración de los productos alimenticios, siendo este un compromiso del hombre para con su salud.

Pocas personas conocen que los alimentos que se consumen todos los días pueden causarle enfermedades conocidas como ETAs (Enfermedades Transmitidas por Alimentos), llamadas así porque el alimento actúa como 
vehículo en la transmisión de organismos patógenos y sustancias tóxicas (Salgado y Castro 2007).

Las ETA están causadas por la ingestión de alimentos y/o agua contaminados con agentes patógenos. Estas enfermedades pueden ser de origen bacteriano, contaminadas con toxinas, entre otros (Flores 2010).

El objetivo de este trabajo es el establecer los controles necesarios que se deben realizar en cada etapa del proceso de producción, con el fin de asegurar que el programa de Buenas Prácticas de Manufactura funcione eficazmente.

Las Buenas Prácticas de Manufactura tienen como objetivo establecer criterios generales de prácticas de higiene y procedimientos para la manufactura de alimentos inocuos, saludables y sanos destinados al consumo humano que hayan sido sometidos a algún proceso industrial. Pero más que esto, deben ser interpretadas como una forma o estilo de trabajo que debe ser conocido y compartido por todos, más allá de los niveles de responsabilidad y calificación técnica. La adopción de las BPM por parte de todos los que participan del proceso productivo contribuye a obtener mayor productividad, a incrementar la seguridad del personal que participa en el mismo, y a mejorar la calidad de los productos, con la consecuente satisfacción del cliente.

\section{Metodología}

El siguiente trabajo se realizó con base en lo que establece el Reglamento de Buenas Prácticas de Manufactura Para Alimentos Procesados, Decreto Ejecutivo 3253, Registro Oficial 696 de 4 de noviembre del 202.

Una acción a la que los países también deben comprometerse es la de mantener el esfuerzo para garantizar la inocuidad tanto de los alimentos que son destinados a la exportación, como aquellos que se asignan al consumo interno, con el firme objetivo de lograr la equidad de acceso a alimentos sanos y aptos para el consumo.

La Organización Mundial de la Salud ha desarrollado las 5 claves de la Inocuidad de los Alimentos, cuya implementación constituyen una accesible manera de evitar las ETA.

Las cinco claves se presentan cada una con una misión especial:

1) Conservar la higiene;

2) Separar alimentos crudos y cocinados;

3) Cocinar completamente los alimentos;

4) Mantener los alimentos a las temperaturas seguras;

5) Usar agua potable y materias primas seguras.

Existen distintas formas en que un alimento puede contaminarse, entre ellas, debido a la presencia de agentes físicos, químicos y biológicos en el producto. 
Como método preventivo, se diseñó un sistema llamado Análisis de Peligros y Puntos Críticos de Control HACCP, para el cual se debe adoptar criterios básicos de control para asegurar la inocuidad de los alimento. Estos son las BPM, las cuales ayudan a evitar la contaminación de los alimentos que comemos. No es un sistema de control de calidad de alimentos, sino que es un sistema preventivo para asegurar la producción de alimentos inocuos. El control disminuye errores en todo el proceso, pudiendo detectarse los mismos en cada una de las etapas.

Según la Fundación Amigos de la Naturaleza (2017), las BPM pueden resumirse en siete importantes factores que determinan la correcta elaboración de los alimentos. Son los que se detallan a continuación:

1. Condiciones higiénico sanitarias de las materias primas.

a) Procedencia de las materias primas: Proveedores calificados; Calidad y origen de las materias primas.

b) Producción: Métodos y procedimientos definidos, revisados y controlados; Identificación clara para evitar contaminación química, física o microbiológica.

c) Almacenamiento: Identificación clara para evitar contaminación química, física o microbiológica y Transporte.

2. Condiciones higiénico sanitarias de los establecimientos elaboradores de alimentos

a) Instalaciones: Diseño, Construcción y Mantenimiento.

b) Higiene de los establecimientos: Conservación, Limpieza y desinfección

c) Subproductos, Manipulación, almacenamiento y eliminación de desechos, Ropa y efectos personales.

3. Recursos humanos

a) Higiene personal: Enseñanza de higiene, Lavado de manos y aseo personal.

b) Estado de salud: Enfermedades contagiosas - heridas

c) Vestimenta: Conducta personal y Visitantes

d) Capacitación y Supervisión

4. Requisitos de higiene en la elaboración

a) Materia prima y otros ingredientes.

b) Prevención de la contaminación cruzada.

c) Empleo del agua.

d) Elaboración.

e) Envasado.

f) Dirección y supervisión.

g) Documentación y registros.

5. Almacenamiento y transporte de materias primas y productos terminados 

a) Identificación clara y visible
b) Evitar la contaminación: cruzada o de otro tipo
c) Registros: humedad y temperatura

6. Controles de proceso de la producción (Controles de laboratorio, procedimientos normalizados)

a) Tipos Analíticos: Químicos, Físicos o Microbiológicos

b) Monitoreo de parámetros: Tiempo, temperatura, humedad, $\mathrm{pH}$, presión

c) Aplicación: A materias primas y otros ingredientes; Durante el proceso de producción y Producto terminado

7. Documentación

a) Procedimientos escritos: Titulo, Objetivo, Alcance, Responsabilidades, Definiciones, Referencias, Instrucciones y Anexos.

Lo que se hizo fue el definir las condiciones sanitarias apropiadas para la manufactura de alimentos de panadería a través de una normativa que permita una producción más segura. Estas disposiciones deben ser cumplidas por el personal operativo y personas que ingresan a la planta, ya sean del departamento administrativo o personas ajenas a la empresa.

Las disposiciones van desde el cuidado que debe tener el personal, los cuidados y medidas preventivas a tomar para la manipulación de los materiales y materias primas antes y durante el proceso así como también la manipulación y cuidados a tomar con el producto terminado, es decir toda la cadena productiva.

a) El Personal.

b) Plantas y terrenos.

c) Disposiciones para áreas externas.

d) Disposiciones para áreas internas.

e) Diseño y construcción de la planta.

f) Paredes puertas, techos y ventanas,

g) Iluminación,

h) Ventilación,

i) Drenajes

j) Medios y controles sanitario, (suministro de agua, almacenamiento de agua, sistemas de drenaje)

k) Instalaciones de baños y vestidores

1) Instalaciones de lavado de manos,

m) Operaciones sanitarias (mantenimiento general, manejo de desechos, control de plagas, medidas de seguridad.

n) Equipo y Utensilios.

o) Controles y procesos.

p) Registros 


\section{Resultados}

Los resultados se obtuvieron luego de plantear un manual de calidad para una planta de panificación y repostería, esto surgió ante la necesidad de optimizar el proceso de producción, pero, sobre todo, para disminuir el número de reclamos realizados por parte de los clientes que se registraron en la empresa.

En base al Manual indicado se realizó una pre-auditoría a dos áreas consideradas críticas debido al impacto que tienen sobre el proceso productivo y el personal de planta.

La metodología empleada para la pre auditoría realizada fue el realizar encuestas con preguntas cerradas (SI) y (NO) para evaluar en cumplimiento de ciertos criterios o principios básicos de calidad para alimentos.

En base al Manual desarrollado como propuesta para ser implementado en la panta de panificación y repostería, se realizó una preauditoría a dos áreas consideradas críticas debido al impacto que tienen sobre el proceso productivo y el personal de planta. A continuación, se presenta el check list para las cláusulas de El Personal y Las Instalaciones de Baños y Vestidores.

Evaluación de "Personal de Trabajo"

\begin{tabular}{|c|c|c|c|}
\hline \multirow{2}{*}{$\mathbf{N}^{\circ}$} & \multirow{2}{*}{ DATOS } & \multicolumn{2}{|c|}{ CUMPLE } \\
\hline & & SI & NO \\
\hline 1 & El personal viste uniforme y ropa adecuada y limpia. & & $\mathrm{X}$ \\
\hline 2 & El personal utiliza correctamente las cofias y mascarillas. & & $\mathrm{X}$ \\
\hline 3 & El personal cuenta con uñas cortas, limpias y sin esmalte. & & $\mathrm{X}$ \\
\hline 4 & El personal no porta joyas dentro del área de producción. & & $\mathrm{X}$ \\
\hline 5 & El personal no ingresa alimentos en el área de producción. & & $\mathrm{X}$ \\
\hline 6 & El personal mantiene limpia su área de trabajo. & & $\mathrm{X}$ \\
\hline 7 & El personal mantiene limpios los utensilios de trabajo. & & $\mathrm{X}$ \\
\hline 8 & $\begin{array}{l}\text { El personal no utiliza celulares o reproductores de música con } \\
\text { auriculares dentro de la planta. }\end{array}$ & & $\mathrm{X}$ \\
\hline 9 & $\begin{array}{l}\text { El personal de despacho cuenta con la protección adecuada para } \\
\text { la manipulación de los alimentos. }\end{array}$ & & $\mathrm{X}$ \\
\hline 10 & El personal se lava las manos al ingresar al área de producción. & $\mathrm{X}$ & \\
\hline 11 & $\begin{array}{l}\text { El personal evita el uso de lapiceros u otros artículos que puedan } \\
\text { afectar la calidad del producto. }\end{array}$ & $\mathrm{X}$ & \\
\hline 12 & $\begin{array}{l}\text { El personal enfermo no se encuentra realizando actividades en el } \\
\text { área productiva. }\end{array}$ & $\mathrm{X}$ & \\
\hline 13 & El personal no utiliza barba y bigote. & & $\mathrm{X}$ \\
\hline 14 & $\begin{array}{l}\text { El personal no coloca ropa y pertinencias personales en lugares } \\
\text { donde los alimentos estén expuestos. }\end{array}$ & & $X$ \\
\hline 15 & El personal no guarda alimentos y bebidas en los casilleros. & $\mathrm{X}$ & \\
\hline
\end{tabular}


Evaluación de "Instalaciones de Baños y Vestidores"

\begin{tabular}{|c|c|c|c|}
\hline \multirow{2}{*}{$\mathbf{N}^{\circ}$} & \multirow{2}{*}{ DATOS } & \multicolumn{2}{|c|}{ CUMPLE } \\
\hline & & SI & NO \\
\hline 1 & $\begin{array}{l}\text { Se cuenta con dispensadores de jabón yodado y alcohol gel en los } \\
\text { baños. }\end{array}$ & $\begin{array}{l}\mathrm{X} \\
\end{array}$ & \\
\hline 2 & $\begin{array}{l}\text { Se cuenta con un instructivo de lavado de manos ubicado en un } \\
\text { lugar visible. }\end{array}$ & & $\mathrm{X}$ \\
\hline 3 & $\begin{array}{l}\text { Se cuenta con dispensadores de toallas desechables y de tachos de } \\
\text { basura. }\end{array}$ & $\mathrm{X}$ & \\
\hline 4 & $\begin{array}{l}\text { Los dispensadores de jabón yodado, alcohol gel y toallas de papel } \\
\text { se encuentran siempre abastecidos. }\end{array}$ & $\mathrm{X}$ & \\
\hline 5 & Los baños cuentan con casilleros en buen estado. & & $\mathrm{X}$ \\
\hline 6 & $\begin{array}{l}\text { Las instalaciones sanitarias se encuentran en buen estado y } \\
\text { cuentan con abastecimiento de agua permanente. }\end{array}$ & $\mathrm{X}$ & \\
\hline 7 & Las instalaciones sanitarias se encuentran limpias y desinfectadas. & & $\mathrm{X}$ \\
\hline 8 & $\begin{array}{l}\text { Los tachos de basura están protegidos por fundas, que son } \\
\text { recogidas periódicamente evitando que se desborden. }\end{array}$ & & $\mathrm{X}$ \\
\hline 9 & Los pisos y paredes se encuentran limpios y desinfectados. & & $\mathrm{X}$ \\
\hline 10 & Los casilleros y vestidores se encuentran limpios y ordenados. & & $\mathrm{X}$ \\
\hline 11 & El techo se encuentra limpio y en buenas condiciones. & & $\mathrm{X}$ \\
\hline
\end{tabular}

Cumplimiento

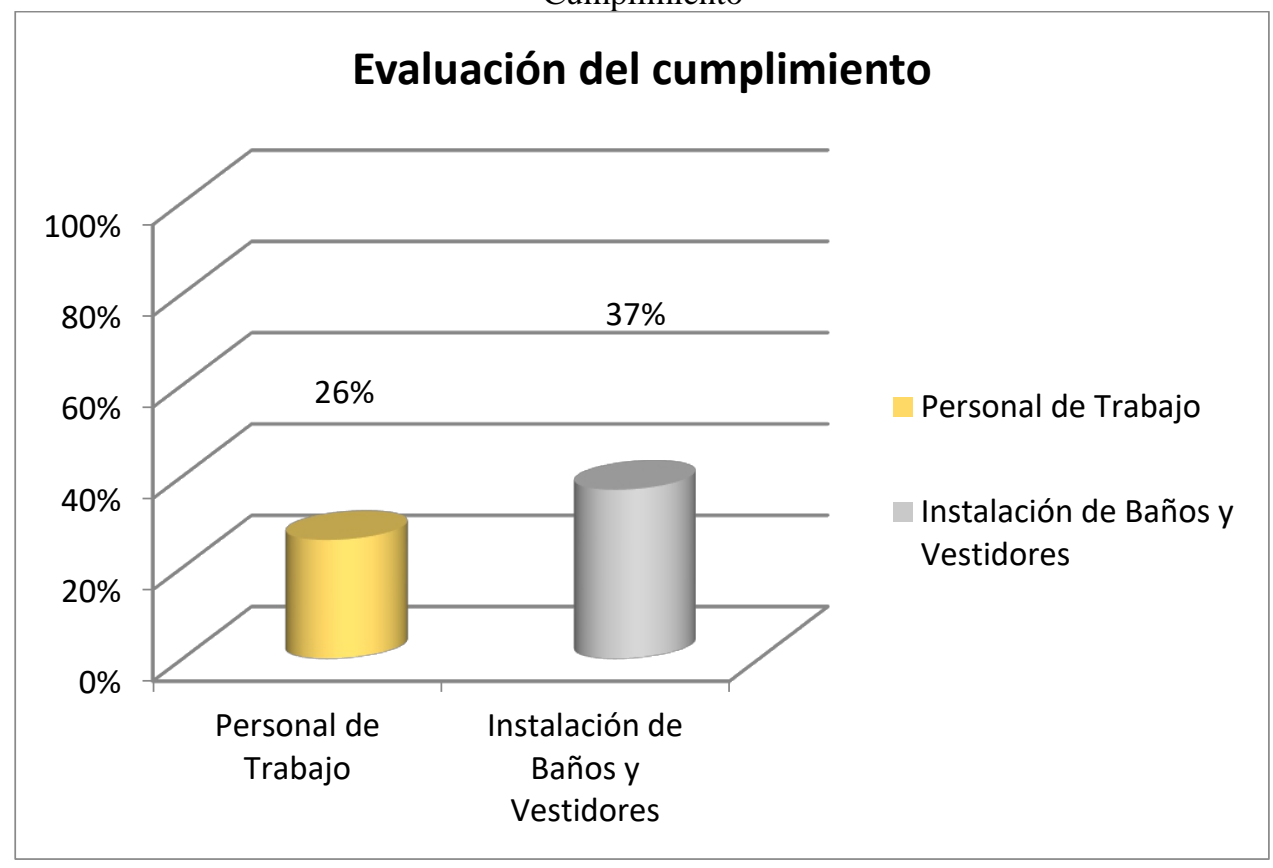




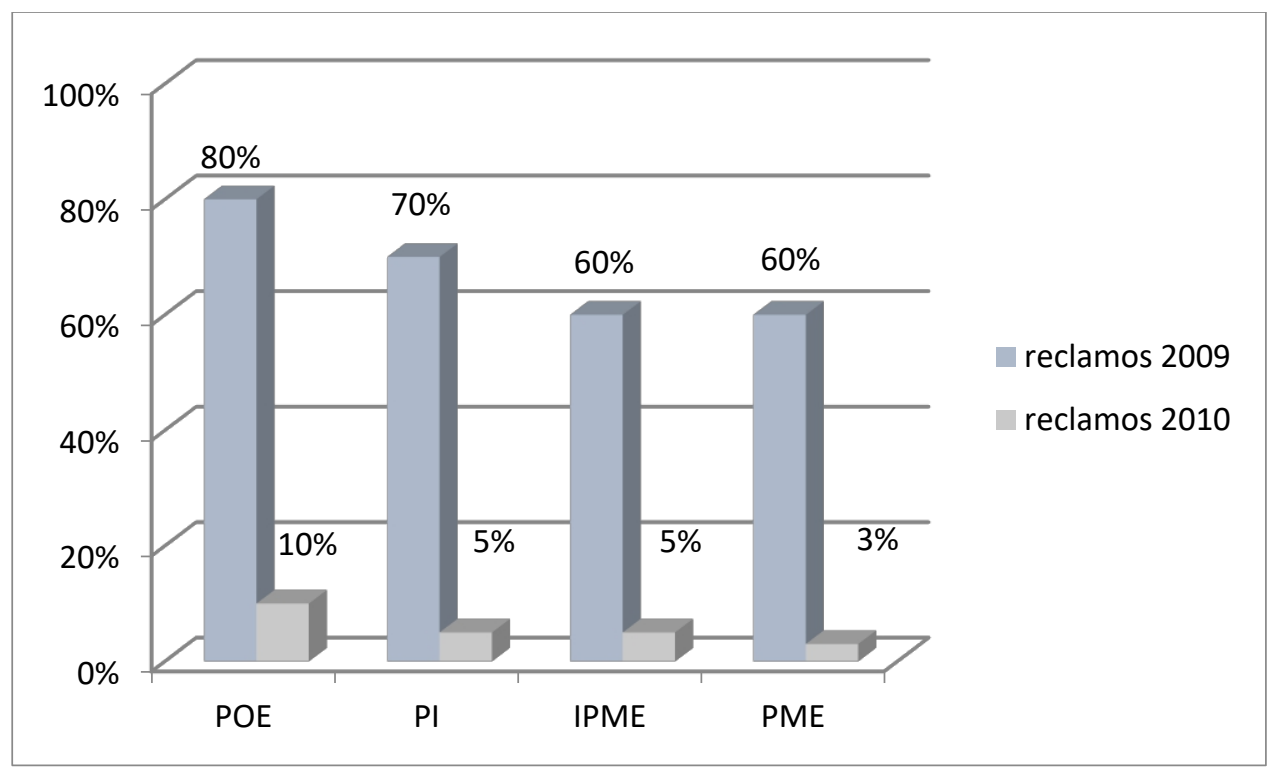

POE $\rightarrow$ Presencia de objetos extraños

PI $\rightarrow$ Presencia de insectos (hormigas, moscas, etc.)

IPME $\rightarrow$ Intoxicación por productos en mal estado

PME $\rightarrow$ Intoxicación por productos en estado de descomposición.

\section{Conclusion}

Se logró impulsar a los integrantes de la empresa a que adopten una cultura de calidad que tomo como base las Buenas Prácticas de Manufactura, estableciendo los controles necesarios que debían ser implementados en cada una de las etapas del proceso de producción de la planta de panificación y repostería, con el fin de asegurar que el programa de Buenas Prácticas de Manufactura funcione eficazmente.

Se aprecia que en el año 2009 se tuvo un considerable número de reclamos por parte de los clientes por distintas razones que se deben a la ausencia de una correcta práctica al momento de la elaboración de los productos. Para el 2010 con la aplicación del manual de Buenas Prácticas de Manufactura propuesto se busca una reducción de los reclamos de los clientes, lo que se vería reflejado como un ahorro de dinero ya que se eliminaría los desperdicios por devoluciones o por materia prima caducada, a su vez se incrementarían las ventas por aumento de fidelidad de los clientes para con la empresa.

Con la pre-auditoría que se realizó, determinó que la empresa tuvo un $26 \%$ de cumplimiento en el área que corresponde a El Personal, y un $36 \%$ en lo que respecta a Instalaciones de Baños y Vestidores. Esto es un problema para la organización, ya que no se está trabajando bajo condiciones adecuadas, lo cual no garantiza un producto que llegue al consumidor con la inocuidad y la seguridad requerida. 


\section{References:}

1. Decreto Ejecutivo 3253. 2002. Reglamento de Buenas Prácticas para Alimentos Procesados. Registro Oficial 696 de 4 de noviembre del 2002.

2. Flores, Carlos E. 2010. «Buenas prácticas de manufactura (BPM)». Revista Electrónica Ingeniería Primero 20: 122-41.

3. Fundación Amigos de la Naturaleza. 2017. Manual de Buenas Prácticas de Manufactura para plantas de transformación de fruto de ASAÏ en pulpa. Santa Cruz de la Sierra, Bolivia.

4. Salgado, M., y K. Castro. 2007. «Importancia de las Buenas Prácticas de Manufactura en cafeterías y restaurantes». Vector 2: 33-40.

5. Sánchez, V., y F. Decker. 2010. «Diseño de un sistema de buenas prácticas de manufactura en una empresa de panificación y repostería y monitoreo de su aplicación vía simulación». Tesis de Magíster en Gestión de la Productividad y la Calidad, Guayaquil-Ecuador: Escuela Superior Politécnica del Litoral. 